\title{
Exploiting Context Information for V2X Dissemination in Vehicular Networks
}

\author{
Michele Rondinone and Javier Gozalvez \\ Uwicore Laboratory \\ University Miguel Hernández \\ Elche, Spain \\ mrondinone@umh.es, \\ j.gozalvez@umh.es
}

\author{
Jérémie Leguay and Vania Conan \\ Advanced Studies Department \\ Thales Communications \& Security \\ Gennevilliers, France \\ jeremie.leguay@thalesgroup.com, \\ vania.conan@thalesgroup.com
}

\begin{abstract}
Cooperative ITS systems are expected to highly improve the efficiency of road mobility. Wireless communications are used by these systems to disseminate centralized real-time traffic information to radio-equipped vehicles. Current proposals for traffic information dissemination either exploit dedicated cellular transmissions to interested vehicles, or cooperatively relay the information through vehicular ad-hoc networks. However, dedicated cellular transmissions may pose energy cost and traffic scalability issues to network operators. On the contrary, purely ad-hoc solutions may suffer from network disconnections and not always ensure adequate service reliability. To overcome these limitations, this paper introduces RoAHD, a hybrid approach in which a few messages injected through the cellular system are followed by a cooperative multi-hop dissemination in the vehicular network. RoAHD exploits multi-hop road connectivity information obtained at a low channel cost. Thanks to this knowledge, it is capable to operate smart injection decisions to ensure good levels of message delivery.
\end{abstract}

Keywords- Cooperative ITS Systems, V2X Data Dissemination, Connectivity Context Awareness

\section{INTRODUCTION}

V2X communications allow the ubiquitous and continuous wireless exchange of information between vehicles (Vehicle-to-Vehicle or V2V), and between vehicles and communications infrastructure nodes (Vehicle-toInfrastructure or V2I). By exploiting these new communications possibilities for the provisioning of advanced safety and real time route planning services, Cooperative Intelligent Transportation Systems (ITS) are paving the way for a major change in vehicular driving experience. $\mathrm{V} 2 \mathrm{~V}$ communications at the 5.8-5.9 GHz band over Vehicular Ad-hoc Networks (VANETs) will be possible thanks to international standards like IEEE 802.11p [1] and its European adaptation ETSI ITS G5 [2]. These standards are readily integrated in system level architectures like the ETSI ITS architecture for Intelligent Transport Systems Communications (ITSC) [3]. Based on these architectures, radio-equipped vehicles, handheld devices, roadside units and traffic management centers will be connected in heterogeneous communications environments also including cellular and broadcast bearers.

The Traffic Management Center (TMC) plays the key role for managing road traffic. Traffic engineers monitor road traffic through inductive loops, sensors, or cameras.
They assess the situation and may decide to change the traffic lights, open signalized corridors, display variable message signs to improve traffic conditions. Cooperative ITS technologies will use vehicles as mobile sensors exploiting V2X communications to produce real-time traffic information and provide the TMC with it. Anomalous events like traffic congestions or accidents detected by vehicles in specific parts of the road network may be of great interest in others distant zones. Cooperative ITS services allowing the TMC to disseminate such up-to-date traffic information over target areas (e.g. Fig 1) will allow drivers to make the best travel decisions.

Nowadays' solutions consider distributing traffic information messages to each vehicle individually using cellular broadband access (e.g. http://waze.com). However, such dissemination schemes may pose energy cost and traffic scalability issues to network operators, currently facing a growing demand of mobile data services. Alternatively, the messages can be cooperatively relayed from vehicle to vehicle (V2V) through the VANET, optionally using opportunistic store, carry and forward techniques [4][5], or leveraging the presence of Roadside Units (RSUs), which can serve as originators of the information, or as fixed relay nodes to passing by vehicles [6][7]. Vehicular mobility poses the main challenge to such $\mathrm{V} 2 \mathrm{~V}$ dissemination strategies as it may induce strong network disconnections. Disconnections may also be caused by insufficient presence of relaying nodes in rural areas, or by the uneven distribution of traffic flows and the obstructing effect of buildings to radio propagation in urban scenarios. All this may impair dissemination's delivery performances over the targeted areas [5]. Opportunistic forwarding techniques can mitigate the negative effects of VANET's disconnections, but generally imply increased delivery delays. If vehicles in distinct and possibly disconnected parts of the targeted area could receive the disseminated message simultaneously and with increased reliability, more drivers would have the chance to analyze it. They could promptly react (e.g. avoiding congested zones), and hence contribute to maximize the traffic efficiency as aimed by the TMC.

Hybrid V2X dissemination strategies combining a few messages injected through the cellular system with VANET's V2V dissemination offer a promising compromise between cellular channel and energy efficiency, and vehicular dissemination effectiveness. First theoretical studies have demonstrated that the best results in this 


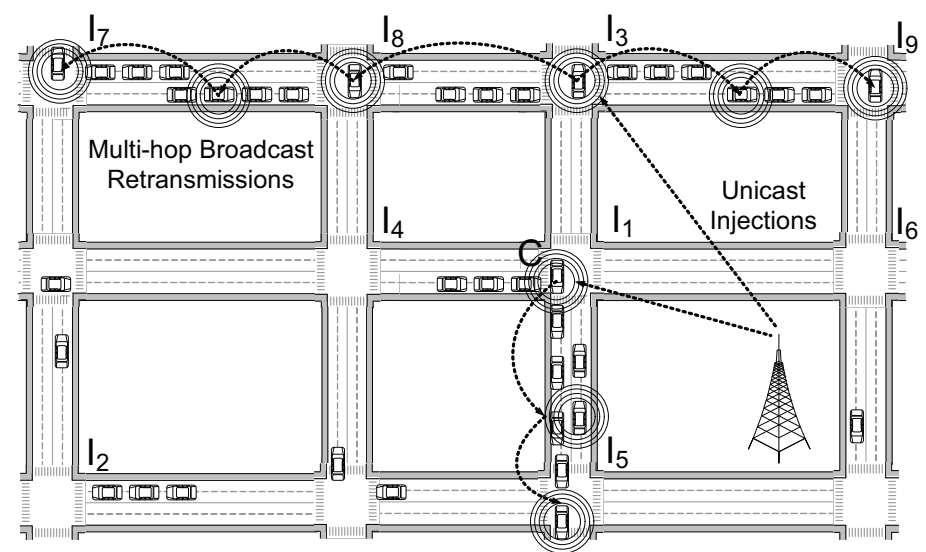

Fig. 1. RoAHD V2X hybrid dissemination scheme.

direction are obtained when the message injection is guided by a global picture of the VANET's V2V connectivity context [8], but no indication is given on how this approach can be optimized to be suitable for a real system. This paper fills a gap in the literature on cooperative ITS systems by proposing RoAHD, a novel scalable and channel-efficient Road Connectivity-Aware Hybrid V2X Dissemination scheme. To comply with cellular channel and energy efficiency, RoAHD considers injecting only a limited number of message copies in the VANET. To ensure that injected messages are reliably disseminated to large sets of recipient vehicles, it exploits VANET's V2V context information. The TMC could derive the spatial distribution of vehicular density by collecting individual vehicle GPS positions through cellular uplink transmissions. However, this could be channel costly for the cellular system, especially in case of high presence of transmitting vehicles. Contrary to this approach, RoAHD builds the V2V context using multi-hop connectivity properties of entire road segments. The multi-hop road connectivity is here defined as the capability of a road segment to support reliable and uninterrupted multi-hop transmissions along its length. As this paper will demonstrate, the multi-hop connectivity of road segments can be measured by vehicles directly in the VANET, and then uploaded to the TMC with a low channel cost. At the TMC, this information is processed and fused to obtain a global connectivity map indicating the road segments that can better support V2V dissemination. By centrally analyzing this map, RoAHD operates smart injection decisions to "seed" the message on VANET's vehicles from where the $\mathrm{V} 2 \mathrm{~V}$ dissemination is expected to reach the largest sets of recipient nodes directly through multi-hop transmissions, and without the assistance of opportunistic forwarding techniques. To implement this dissemination, RoAHD defines a particular multi-hop broadcasting protocol providing the message with the maximum penetration over any possible direction of the targeted area.

We compare RoAHD's approach with other hybrid dissemination solutions deriving $\mathrm{V} 2 \mathrm{~V}$ connectivity out of individual vehicle positions. The obtained simulation results demonstrate that RoAHD generates a trustful measure of the actual VANET's V2V dissemination capabilities, and hence results in injections ensuring good delivery performance. More interestingly, this performance is obtained with a lower channel cost on the cellular system, and a negligible impact in the vehicular ad-hoc network.

The paper is organized as follows. Section II introduces the RoAHD proposal. Section III and IV respectively present the methodologies used to generate, collect and process the multi-hop road connectivity information needed to derive RoAHD's connectivity context characterization. In Section $\mathrm{V}$, an overview of RoAHD's injection strategy and V2V dissemination scheme is given. After outlining in Section VI the conducted performance evaluation, Section VII will present the related studies, and Section VIII will conclude this work.

\section{ROAHD PRINCIPLE}

RoAHD defines a V2X hybrid dissemination scheme to deliver traffic efficiency messages to the vehicles belonging to a target area (Fig. 1). Without loss of generality, the UMTS cellular technology is considered in this work as the infrastructure-based communications system adopted by the TMC to communicate with vehicles. The TMC uses UMTS downlink transmissions to simultaneously inject message copies to specific injection vehicles in the VANET. In turn, the injection vehicles start to disseminate the message in the VANET using V2V multi-hop broadcast retransmissions (Fig. 1). RoAHD's goal is to reduce the cellular system's channel and energy consumption by using only a limited number of injected messages, while maximizing the message delivery resulting from $\mathrm{V} 2 \mathrm{~V}$ dissemination over the target area. To achieve this objective, a global knowledge of the VANET's V2V connectivity context is needed. Through such context characterization, the TMC would learn where the injected messages could be safely multi-hop rebroadcasted and delivered to a high number of vehicles in the VANET. As a result, it could implement injection strategies to address large sets of recipient nodes with only a few messages smartly injected through the cellular system. 


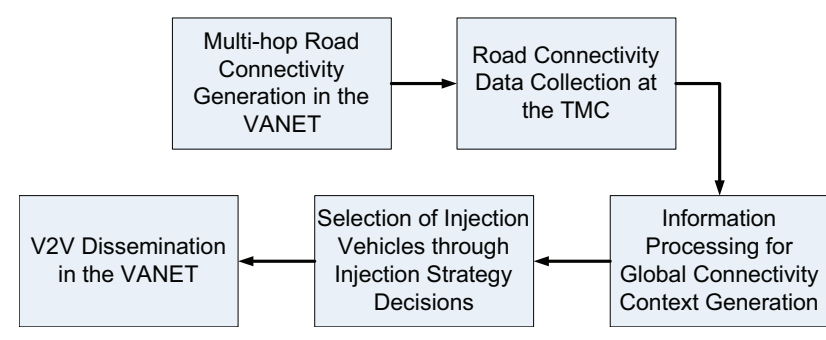

Fig. 2. RoAHD's Operational Functioning.

In previous studies, the TMC obtains a VANET's V2V connectivity picture by collecting individual vehicles' information such as GPS positions or lists of neighbors [8] [9]. However, if every vehicle was involved in the periodic upload of such information, the scalability of the system might be compromised, which could in turn provoke non negligible effects on the other services [10]. Contrary to these schemes, in RoAHD the TMC collects information about multi-hop road connectivity. The multi-hop connectivity measures the capability of a road segment to support uninterrupted multi-hop transmissions, and reflects the presence of adequately distributed vehicles along its length (Fig. 3). In a VANET, the multi-hop road connectivity can be assessed in real-time by running the distributed and lightweight V2V DiRCoD protocol [11]. The DiRCoD multi-hop road connectivity information is "sensed" by vehicles placed at road intersections (in the example of Fig.1, vehicle $\mathrm{C}$ at intersection $\mathrm{I}_{1}$ would be informed about the possibility to relay a message to intersection $\mathrm{I}_{5}$ directly through multi-hop transmissions). Considering this, only vehicles at road intersections are in charge of regularly uploading information to the TMC, which permits RoAHD to save UMTS uplink channel resources. At the TMC, the DiRCoD connectivity information of every road segment is processed and fused to derive a global V2V multi-hop road connectivity map. By analyzing the multi-hop connectivity of the roads between adjacent intersections, the TMC computes sets of connected intersections through which a message copy would be safely multi-hop rebroadcasted (e.g. the set of intersections $I_{7}, I_{8}, I_{3}$, and $I_{9}$ in Fig. 1). Moreover, the TMC observes to which extent the multi-hop connectivity of road segments is stable over time to derive indications about the density of vehicles. In fact, roads providing multi-hop connectivity for extended periods indicate a higher presence of vehicles. Based on this context characterization, RoAHD defines a message injection strategy aiming at injecting a limited number of message copies over the road segments with the highest connectivity stability to address the largest sets of possible recipient nodes. Message copies are injected over vehicles that start disseminating in the VANET with an optimized multi-hop broadcasting protocol in which only a subset of receivers is in charge of retransmitting them (Fig. 1).

A flow diagram summarizing all the steps followed by RoAHD's operational functioning is depicted in Fig 2.

\section{ROAD CONNECTIVITY ESTIMATION AND COLLECTION}

This section describes how the road connectivity information used by RoAHD is assessed in the VANET and successively collected at the TMC.

\section{A. DiRCoD Connectivity Estimation}

DiRCoD [11] exploits V2V communications to estimate the multi-hop connectivity of a road segment and notify this information to the intersections that delimit it. In particular, it relies on standard broadcast beacon messages periodically transmitted by vehicles to inform neighboring nodes of their geographical position. Let it be considered that the vehicle $\mathrm{E}$ in Fig. 3 has to be informed about the multi-hop connectivity of the road segment in the direction $\mathrm{I}_{1} \rightarrow \mathrm{I}_{2}$. The road segment is defined to be fully connected in this direction if it contains a sufficient number of spatially distributed vehicles to multihop forward a message from $I_{1}$ to $I_{2}$ without interruptions (Fig. 3a). On the contrary, the road segment is partially connected if a message transmitted from $\mathrm{I}_{1}$ would only reach a vehicle placed at a given distance from $\mathrm{I}_{2}$ (Fig. 3b). To quantify this remaining distance, and thereby the connectivity status of the road segment, DiRCoD defines the Virtual Distance (VD) metric separating $\mathrm{I}_{2}$ from $\mathrm{I}_{1}$ : the lower the VD, the better the multi-hop connectivity. To estimate the VD, DiRCoD divides the road into road sections numbered with increasing values as their distance to $I_{2}$ increases (see Fig. 3) and with a length equal to the vehicles' average communications range. DiRCoD defines the VD separating $\mathrm{I}_{1}$ from $\mathrm{I}_{2}\left(V D_{12}\right)$ as the number of road sections (or hops) between $I_{2}$ and the closest vehicle to $I_{2}$ that can be reached from $\mathrm{I}_{1}$ through multi-hop transmissions. In the case of Fig. 3b (partial multi-hop road connectivity), $V D_{12}$ is 2 hops since a message transmitted from $\mathrm{I}_{1}$ can only reach vehicle B placed at 2 hops distance from $\mathrm{I}_{2}$. On the contrary, in Fig. 3a (full multi-hop road connectivity), $V D_{12}$ is 0 since the message can reach $I_{2}$ directly through multi-hop

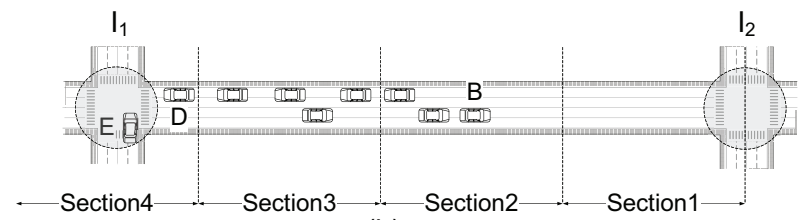

(b)

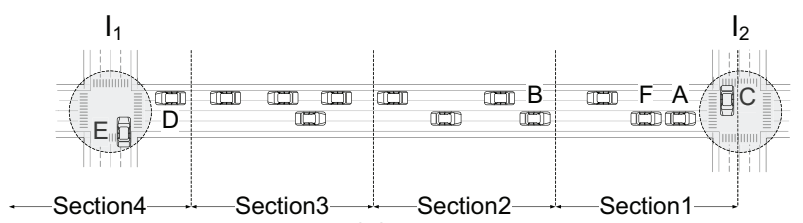

(a)

Fig. 3. DiRCoD's full (a) and partial (b) multi-hop road connectivity. 
transmissions.

The DiRCoD's VD is included in a Connectivity Field (CF) appended by vehicles to standard beacons. A vehicle appends to its beacon a CF indicating the road section it is placed at, unless it detects (by consulting its neighbor table) that other vehicles are closer to $\mathrm{I}_{2}$. Referring to Fig. $3 \mathrm{~b}$, vehicle $B$ includes a $C F$ indicating a VD of ' 2 ' in its beacon. On the contrary, vehicle $F$ in Fig. 3a (that would initially append ' 1 ' to its beacon) appends a CF indicating ' 0 ' hops to $\mathrm{I}_{2}$ only upon receiving a beacon from vehicle $\mathrm{C}$. The $\mathrm{CF}$ is then forwarded towards $\mathrm{I}_{1}$ by the other vehicles along the road. These vehicles activate a distributed contention-based mechanism to select the vehicles that include the overheard $\mathrm{CF}$ in their beacon. As a result, vehicles placed at $\mathrm{I}_{1}$ receive a beacon with a CF of ' 0 ' in Fig. 3a, and a CF of ' 2 ' in Fig. 3b. DiRCoD also defines a method to control the period between two consecutive road connectivity assessments. Such period is referred to as $C F$ generation period, and indicates the time that vehicles have to wait before generating or forwarding new CFs. In previous works it has been demonstrated that a $\mathrm{CF}$ generation period of $2 \mathrm{~s}$ is enough to follow the time variation of road segments' connectivity status [12] Considering in this work a $\mathrm{CF}$ generation period of $2 \mathrm{~s}$, if a road segment is fully of partially connected, vehicles at intersections receive DiRCoD CFs every $2 \mathrm{~s}$.

\section{B. DiRCoD Information Collection}

RoAHD defines a Cellular Intersection-based (CI) Uploading scheme for DiRCoD's connectivity information collection at the TMC. A vehicle uploads a DiRCoD Connectivity Update (DCU) after crossing the center of an intersection $\mathrm{I}_{\mathrm{i}}$. The DCU contains the DiRCoD information of all $\mathrm{I}_{\mathrm{i}}$ 's adjacent road segments. More precisely, the DCU includes the virtual distances $V D_{i j}$ separating $\mathrm{I}_{\mathrm{i}}$ from all its adjacent intersections $\mathrm{I}_{\mathrm{j}}$. These $V D_{i j}$ are overheard by the vehicle crossing $I_{i}$ by receiving beacons with appended DiRCoD's $C_{i j}$ (CFs referring to road segments $I_{i} \rightarrow I_{j}$ ). Before uploading a DCU, the vehicle checks, for all the adjacent intersections $\mathrm{I}_{\mathrm{j}}$ whether it received a $\mathrm{CF}_{\mathrm{ij}}$ within the last $\mathrm{CF}$ generation period seconds. The absence of $\mathrm{CF}_{\mathrm{ij}}$ receptions in this period indicates that the intersection $\mathrm{I}_{i}$ is currently separated form $\mathrm{I}_{\mathrm{j}}$ by a virtual distance of $V D_{i j m a x}$ hops (e.g. 4 for the road segment depicted in Fig. 3). As a consequence, the vehicle includes a $V D_{i j}$ equal to $V D_{i j \max }$ in the DCU. To prevent neighboring vehicles from uploading a DCU for intersection $I_{i}$ in the very next instants (thereby avoiding wasting uplink channel resources), the vehicle transmits a beacon including an Uploading Field (UF). Vehicles receiving this field activate a timer of $T_{U}$ seconds (uploading timer duration) during which prospective DCU uploads for $\mathrm{I}_{\mathrm{i}}$ are disabled. As a result, $T_{U}$ is a protocol parameter that can be configured to control the period between consecutive uploaded DCUs.

\section{Global Connectivity Context Generation}

RoAHD aims at detecting road segments with high multihop road Connectivity Stability (CS) over time. Multi-hop $\mathrm{CS}$ can in fact return indications on the possibility for a given road segment to maintain connectivity in the next instants given that it has been connected in the last time period. This is very important, given that message injections from the TMC to the VANET can be performed with a given delay compared to when the connectivity information is uploaded. Moreover, in most of the cases, a road providing high CS indirectly indicates a high presence of vehicles on it. Hence, this information can be exploited by injection strategies. Injection strategies can be defined as procedures aimed at selecting a "strategic" combination $V^{n}$ of $n$ injection vehicles that is expected to optimize the performance of the dissemination. For the dissemination of traffic efficiency messages considered in this work, the main objective is to reach the highest number of recipient vehicles over a target area. Considering this, the context characterization achieved in terms of connectivity stability is used by RoAHD to derive the Coverage Level $C L\left(V^{n}\right)$, an indicator of the expected amount of vehicles that would be reached after injecting message copies on a combination of vehicles $V^{n}$ : the higher the CL, the more effective the injection. Since RoAHD considers the use of a limited number $\underline{n}$ of injected message copies, its injection strategy is aimed at identifying the combination of $\underline{n}$ injection vehicles that maximizes the $C L\left(V^{\underline{n}}\right)$.

The TMC runs a connectivity processing scheme to derive the connectivity stability of all the road segments in the target area out of the DCUs collected at different instants and from different intersections. The connectivity processing scheme exploits this information to calculate a global V2V connectivity map and estimate the expected coverage level that drives its message injection strategy.

\section{A. Connectivity Stability Computation}

The connectivity processing scheme computes connectivity stability values $C S_{V D i j}(t)$ as an estimation of the percentage of time in which the road segment $I_{i} \rightarrow I_{j}$ experiences a specific virtual distance $V D_{i j}=\mathrm{VD}$. Connectivity stability values $C S_{V D i j}(t)$ are computed for all the possible values VD in the interval $\left[0,1, \ldots,\left(V D_{\text {ijmax }}+1\right)\right]$ that road segment $\mathrm{I}_{\mathrm{i}} \rightarrow \mathrm{I}_{\mathrm{j}}$ can experience. According to Section III.A, $V D_{i j}=0$ indicates that the road experiences a full multi-hop road connectivity status, and $0<V D_{i j}<V D_{i j \max }$ partial connectivity status. $V D_{i j}=V D_{i j m a x}+1$ (e.g. 5 for the road segment depicted in Fig. 3) indicates a status of absence of connectivity, that is a situation in which there is no vehicle at intersection $\mathrm{I}_{\mathrm{i}}$ to upload a DCU. The connectivity stability values are computed and updated at regular time steps of $1 \mathrm{~s}$ based on $V D_{i j}$ values contained in collected DCUs. Let us consider that a DCU containing $V D_{15}=0$ (full connectivity over road $I_{1} \rightarrow I_{5}$ in Fig. 1) is uploaded by vehicle $C$ at a given instant. Since the processing scheme expects receiving DCUs at regular intervals of $T_{U}$ seconds, it stores $V D_{15}=0$ for at most the next $T_{U}$ time steps. If a new DCU is received by $T_{U}$ seconds from the last update, the processing scheme starts to store the $V D_{15}$ contained in it, otherwise it assigns $V D_{15}$ a default value of $V D_{15 \max }+1$ (the connectivity processing scheme considers that $I_{1} \rightarrow I_{5}$ has become disconnected). To compute the connectivity stability values $C S_{V D 15}(t)$, the processing scheme considers the last $T_{C S}$ values of $V D_{15}$ stored. Over a generic road segment $\mathrm{I}_{\mathrm{i}} \rightarrow \mathrm{I}_{\mathrm{j}}$, the connectivity 
stability $C S_{V D i j}(t)$ of $V D_{i j}=\underline{\mathrm{VD}}$ is computed as the ratio between the number of time steps in which $V D_{i j}=\mathrm{VD}$ over the last $T_{C S}$ time steps, and $T_{C S}$. In order to provide $\overline{C S}_{V D i j}(t)$ with a statistical relevance, the considered $T_{C S}$ is set to $90 \mathrm{~s}$ in this work.

\section{B. Coverage Level Estimation}

The V2V context characterization described in section IV.A is based on the expected stability of multi-hop road connectivity statuses measured by vehicles at road intersections. Considering this, RoAHD restricts the injection of message copies only to vehicles placed at intersections. The connectivity processing scheme computes the coverage level $C L_{i}(t)$ of every intersection $\mathrm{I}_{\mathrm{i}}$ to achieve an indication of the amount of vehicles that could be reached if a message copy was injected there. The $C L_{i}(t)$ at intersection $\mathrm{I}_{\mathrm{i}}$ is computed as the sum of the coverage levels $C L_{i j}(t)$ of every adjacent road segment $I_{i} \rightarrow I_{j}$ of $I_{i}$. Over a generic road segment $\mathrm{I}_{\mathrm{i}} \rightarrow \mathrm{I}_{\mathrm{j}}, C L_{i j}(t)$ can be assigned continuous values in the interval $\left[0,\left(V D_{i j \max }+1\right)\right]$. A graphical representation of the coverage level computation over two adjacent intersections is depicted in Fig. 4.

For the calculation of the $C L_{i j}(t)$ of a road segment, some further definitions are needed. A road segment $I_{i} \rightarrow I_{j}$ instantaneously holds a Coverage Range $C_{i j}(t)$ defined as the complement of the measured DiRCoD's virtual distance: $C_{i j}(t)=\left(V D_{i j \max }+1\right)-V D_{i j}(t)$. According to this, the lower the instantaneous $V D_{i j}(t)$, the higher the coverage range. Let $c_{V D i j}$ indicate the coverage range associated to a given value VD of the virtual distance $V D_{i j}$ measurable on a specific road segment $\mathrm{I}_{\mathrm{i}} \rightarrow \mathrm{I}_{\mathrm{j}} \quad$ (e.g. the road segment of Fig. 3 has $\left(V D_{12 \max }+1\right)=5$, thus the coverage range associated to $V D_{12}=0$ is $c_{0}=5$; the coverage range associated to $V D_{12}=1$ is $c_{1}=4$, and so on). According to these definitions and those of Section IV.A, an association exists between $V D_{i j}, c_{V D i j}$, and $C S_{V D i j}$, for any measurable $\underline{\mathrm{VD}}$ over a given road $\mathrm{I}_{\mathrm{i}} \rightarrow \mathrm{I}_{\mathrm{j}}$. In this context, the instantaneous coverage level $C L_{i j}(t)$ is computed as a weighted average of all the measurable coverage ranges $c_{V D i j}$ of road segment $\mathrm{I}_{\mathrm{i}} \rightarrow \mathrm{I}_{\mathrm{j}}$ :

$$
C L_{i j}(t)=\frac{1}{\sum_{V D_{i j}=0} w_{V D i j}(t) C S_{V D i j}(t)} \sum_{V D_{i j}=0}^{V D_{i j \max }+1} w_{V D i j}(t) C S_{V D i j}(t) c_{V D i j}
$$

The computed $C L_{i j}(t)$ has continuous values in the interval $\left[0,\left(V D_{i j m a x}+1\right)\right]$. The coverage ranges $c_{V D i j}$ are weighted by the currently experienced connectivity stability values $C S_{V D i j}(t)$ associated to $V D_{i j}=\mathrm{VD}$. Since the $C S_{V D i j}$ values are calculated over a moderately long observation window ( $T_{C S}=90 \mathrm{~s}$ in this work), they slowly adapt to changes of the connectivity status of a road segment. As a consequence, instantaneous $C S_{V D i j}(t)$ values might not perfectly represent current road's connectivity and coverage capabilities. To cope with this issue while preserving the statistical relevance achieved from CS values, the $C L_{i j}(t)$ calculation (1) also includes the weights $w_{V D i j}(t) . w_{V D i j}(t)$ are used to weight a $C S_{V D i j}(t)$ according to the time $T_{\underline{V D}}(t)$ passed from when the connectivity processing scheme last assigned the value $\underline{\text { VD }}$ to $V D_{i j}$ (see Section IV.A). The weigths $w_{V D i j}(t)$ have continuous values in the interval $[0,1]$ and are built to exponentially decay as $T_{\underline{V D}}(t)$ increases. As a result, the connectivity stability associated to $\underline{\mathrm{VD}}$ values that have not been recently detected by the processing scheme has less influence in the $C L_{i j}$ calculation.

\section{Message InJECtion AND V2V Dissemination}

To select the combination $V^{n}$ of injection vehicles maximizing the overall coverage level $C L\left(V^{n}\right)$, the TMC's connectivity processing scheme updates at every time step the expected coverage level $C L_{i}(t)$ of every intersection $\mathrm{I}_{\mathrm{i}}$. Moreover, according to Section III.A, if a road segment $I_{i} \rightarrow I_{j}$ holds good connectivity, then a message copy injected at $I_{i}$ can be multi-hop disseminated to $I_{j}$, and from $I_{j}$ over the road segments that are adjacent to it. Considering this, the connectivity processing scheme also computes Connected Sets of Intersections (CSIs). Injecting one message over any of the intersections composing a CSI would be enough to disseminate the message over all the road segments of the connected set by V2V retransmissions. In this work, two intersections $\mathrm{I}_{\mathrm{i}}$ and $\mathrm{I}_{\mathrm{j}}$ form a CSI at instant $t$ if the road segment between them is assigned a CL higher than a given threshold (set in this work to $80 \%$ of $V D_{i j \max }+1$ ) over both its directions:

$$
C L_{i j}(t) \geq T h r \cap C L_{j i}(t) \geq T h r
$$

Injecting on a CSI is expected to ensure a coverage level $C L_{C S I}$ equal to the sum of the $C L_{i}$ of the intersections

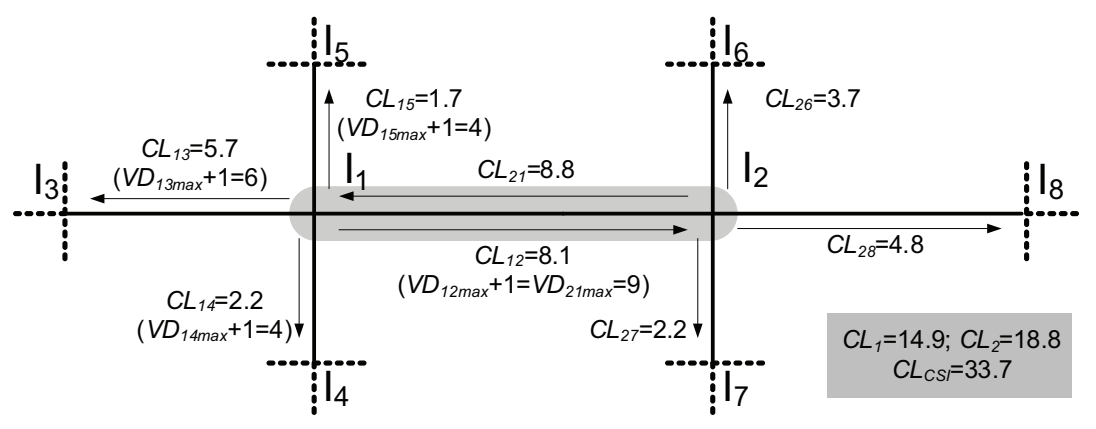

Fig. 4. Coverage level over a connected set of intersections. 
composing the connected set (e.g. in Fig. $4, I_{1}$ and $I_{2}$ form a CSI).

Based on these definitions, when a message injection is needed, the connectivity processing scheme computes connected sets of intersections CSIi, and calculates their expected coverage level $C L_{C S I i}$ (intersections not belonging to any CSIi are treated as CSI of size equal to 1). To obtain the maximum CL with a limited number of message copies, the available $\underline{n}$ copies are injected in the $\underline{n}$ CSIs with the highest expected $C L_{C S I i}$. On a given CSIi, the copy is injected on a vehicle placed at the intersection providing the highest $C L_{i}$, given that (according to the CL and CS definitions) it is expected to be crossed by the highest flows of vehicles. The injection vehicle is the one that last uploaded a DCU for $\mathrm{I}_{\mathrm{i}}$. This injection strategy will be referred to in the following as Injection with Intersection-based Road Connectivity (IRC) Characterization Awareness.

Injecting message copies at road intersections also optimizes the V2V dissemination in the VANET. Injection vehicles can exploit Line-of-Sight (LOS) propagation conditions towards various road segments to simulateneously disseminate the message to all the vehicles placed over them. In this context, RoAHD defines and adopts a V2V dissemination mechanism using multi-hop broadcast retransmissions in which a limited set of rebroadcasters are selected in a distributed fashion according to their capability to provide the message with the maximum progress in any possible direction. To achieve this, a distributed contentionbased algorithm is implemented. Based on this algorithm, receiving nodes that are more distant from the broadcasters, as well as nodes that are closer to the centers of still unaddressed road intersections are selected for retransmission (Fig. 1).

\section{PERformance EVAluation}

To demonstrate the channel efficiency and delivery effectiveness of RoAHD, this work considers its comparison with other hybrid dissemination approaches. These approaches inject the same number $\underline{n}$ of message copies in the VANET and adopt the same V2V dissemination scheme as RoAHD. However, they inject messages based on different VANET connectivity characterizations, which results in the definition of the following strategies:

1) Injection with Uploaded Vehicle GPS Positions Connectivity Characterization Awareness (in the following referred to as "GPS"). As defined in other works ([8][9]), a centralized V2V connectivity characterization is obtained by combining individual vehicle GPS positions uploaded every $10 \mathrm{~s}$. This uploading period is higher than the $2 \mathrm{~s}$ used in [9], and lower than the $60 \mathrm{~s}$ defined in [8]. In this work, a $10 \mathrm{~s}$ uploading period has been selected after verifying the best tradeoff between connectivity characterization's precision and required uplink channel cost. Analyzing the vehicle GPS positions, the TMC derives Connected Sets of Vehicles (CSVs) in the VANET, i.e. sets of vehicles that can communicate with direct or multi-hop transmissions. For this purpose, a deterministic communications range model is used according to which any two vehicles are connected in the VANET if they are within each others' communications ranges and in LOS conditions (it is considered that the processing scheme holds a digital map of the buildings of the scenario). The $\underline{n}$ available message copies are injected in the $\underline{n}$ CVSs with the highest number of vehicles. In a CSV, the message is injected on the vehicle having the shortest average distance from the other vehicles of its CSV.

2) Injection with Actual Vehicle Positions Connectivity Characterization Awareness. This injection strategy adopts the "idealistic" V2V connectivity characterization that the TMC would have if it knew the actual positions of vehicles at every moment. At the moment of injecting, the TMC retrieves these positions from the adopted simulation environment, and calculates CSVs and injection vehicles as explained for the injection startegy based on GPS charachterization. Since this charaterization is not realistic, the associated injection startegy is used as an idealistic benchmarck to compare the performance of the other injection schemes.

3) Random Injection. This injection strategy adopts no connectivity characterization. The $\underline{n}$ available message copies are injected on $\underline{n}$ distinct randomly selected vehicles. This strategy permits quantifying the added value, in terms of delivery performance, that considering a V2V connectivity characterization provides to a hybrid V2X dissemination scheme.

\section{A. Evaluation Environment}

The above described performance evaluation has been investigated through simulations on the ETSI ITSC standardcompliant iTETRIS simulation platform [13]. Thanks to its unique architecture integrating the ns-3 network simulator (http://www.nsnam.org/), the SUMO traffic simulator (http://sumo.sf.net/), and the language-agnostic implementation of any cooperative ITS application (iAPP), iTETRIS is capable to simulate the mutual dependence between vehicular mobility, wireless communications, and cooperative ITS services in a highly modular way. Vehicles' mobility is simulated in SUMO; DiRCoD's transmissions, DCU and vehicle GPS position uploads in ns-3. The TMC's connectivity processing scheme for connectivity context characterization and message injection decisions is simulated on the iAPP.

Without any loss of generality, the adopted SUMO vehicular traffic scenario is a Manhattan-like grid over an area of $1.7 \times 1.3 \mathrm{~km}$. The grid is composed by 30 intersections and 98 road segments with different lengths (ranging from $200 \mathrm{~m}$ to $450 \mathrm{~m}$ ) and vehicular traffic densities (from 0 to 32 vehicles $/ \mathrm{km} / \mathrm{lane}$ ). Time and space variations of the vehicular traffic flows are imposed over the Manhattan scenario in order to verify the capability of the compared dissemination schemes to react to changes in the overall V2V connectivity configuration.

Every simulated vehicle is able to communicate using a UMTS UE and a $5.9 \mathrm{GHz}$ ETSI ITS G5 radio interface. Every ITS G5 interface broadcast standard beacon messages with a $2 \mathrm{~Hz}$ frequency, transmitting with $6 \mathrm{Mbit} / \mathrm{s}$ data rate and using a transmitting power of $20 \mathrm{dBm}$. Given the significant impact of radio propagation on wireless communications, propagation conditions including pathloss, 
shadowing and multipath fading are modeled using the urban micro-cell propagation model for the $5 \mathrm{GHz}$ band developed by the European project WINNER [14], and included in iTETRIS. With the adopted transmitting power and propagation model, an inter-vehicle average packet reception rate of $99 \%$ is experienced between two vehicles separated by $95 \mathrm{~m}$ and communicating in LOS conditions. Considering this, an average communications range of $95 \mathrm{~m}$ has been used to set the length of the DiRCoD's road sections. The same communications range value is used to compute the connected sets of vehicles (CSVs) of the dissemination schemes based on vehicles positions connectivity characterizations.

To support the collection of DCUs and vehicle positions, as well as the injection of traffic efficiency messages, a UMTS node B serving the whole considered area is deployed. The amount of information carried by an uploaded DCU at intersection $I_{i}$ is 8 bytes to code the intersection position, plus one byte to represent the virtual distance $V D_{i j}$ measured over the adjacent road segment $\mathrm{I}_{\mathrm{i}} \rightarrow \mathrm{I}_{\mathrm{j}}$, for each of the $V D_{i j}$ contained in the message. On the contrary, to upload a vehicle's position 8 bytes are needed. Due to the small size of these messages, this work considers their transmission on the UMTS Random Access Channel (RACH) mapped on the Physical RACH (PRACH). The RACH is a common uplink channel normally used for signaling purposes. Anyways, its adoption for transmissions of small amount of data can increase the number of simultaneous connections given that users are not required to activate dedicated channels. The feasibility of frequent (every 10s) vehicular data uploading on the PRACH is studied in [10]. As the authors indicate, if the uploaded message is adequately small ( 20 bytes on $10 \mathrm{~ms}$ frames), up to 600 users per cell can be served. To calculate the overhead generated on the PRACH by the compared uploading schemes, this work considers transmitting uplink messages in 128 bits (16 bytes) Radio Link Control (RLC) payloads over frames of $10 \mathrm{~ms}$. As described in [15], this transmission mode implies a user data rate of $12.8 \mathrm{kbps}$, and a total overhead (considering the additional overhead of the lower layers) of 388 bits ( 48.5 bytes) per uploaded message. In phase of injection, the UMTS node B transmits message copies of the same traffic efficiency message using dedicated channels with a user data rate of $128 \mathrm{kbps}$. Injected messages copies have a payload of 300 bytes. Injections are performed periodically every $10 \mathrm{~s}$ for an overall simulation period of $1000 \mathrm{~s}$. The simulation results reported in the following provide an accuracy equivalent to relative errors below 0.05 .

\section{B. Simulation Results}

We first evaluate the channel efficiency of the investigated hybrid dissemination schemes by comparing the amount of information collected on the UMTS uplink channel, and required to build a specific VANET's V2V connectivity characterization. In this context, Fig. 5a shows the instantaneous UMTS uplink overhead generated by the vehicle positions ("VP") uploading scheme (needed for the GPS characterization), and by RoAHD's cellular intersection-based road connectivity uploading mechanism with a $T_{U}$ of $5 \mathrm{~s}$ ("CI5", needed for RoAHD's IRC characterization). According to their definitions, the dissemination based on random injections and the dissemination with the idealistic connectivity characterization awareness do not generate such overhead. As Fig. 5a shows, the VP uploading scheme's overhead is much more dependent on the total number of vehicles present in the road network compared to that generated by the CI uploading scheme (in the central period of the simulation time a higher number of vehicle is present). As a consequence, RoAHD's CI uploading technique demonstrates a better scalability. This lower overhead is due to the fact that, since DCUs are only uploaded at road intersections, their number is less dependent on the amount of vehicles, and only varies as a function of the number of intersections in the scenario. Throughout the whole simulation time, the CI uploading mechanism generates a cumulated UMTS uplink overhead more than 6 times smaller than that produced by the VP uploading scheme. It is important to remind that, although being more efficient in the use of the UMTS uplink channel, the CI uploading scheme also requires generating overhead in the VANET. This overhead is due to the additional connectivity fields (CFs) and uploading fields (UFs) included in standard beacon messages respectively by the DiRCoD mechanism and by the CI uploading scheme (See Section III). However, both CFs and UFs require only one additional byte information. As a result, and as depicted by Fig. 5b), the instantaneous additional overhead generated in the VANET by the CI scheme is limited if compared to that created on the UMTS uplink channel by the VP uploading scheme, and has less relevance if considering the higher ITS G5's data rates.

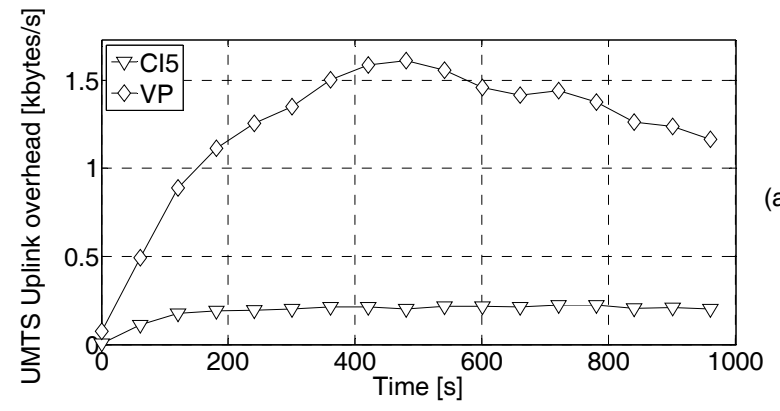

(a)

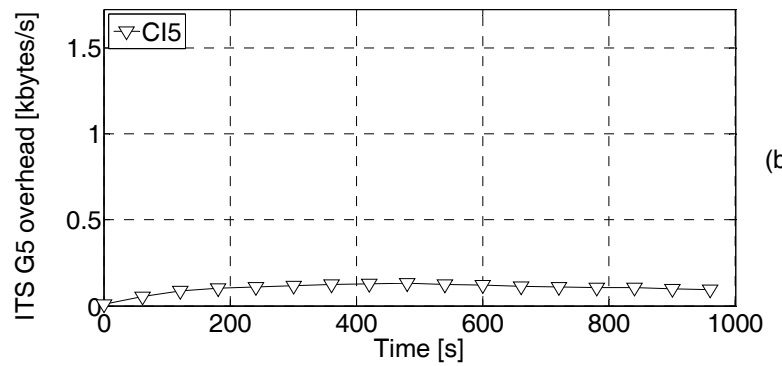

Fig. 5. Instantaneous overhead needed for the VANET's V2V connectivity characterization of the compared dissemination schemes.

The previous results demonstrate that RoAHD's CI uploading scheme can leverage the generation of a global VANET connectivity characterization with important savings in the cellular uplink channel utilization. To 
demonstrate that this less channel consuming connectivity characterization can successfully support effective and efficient hybrid dissemination schemes, the results of Fig. 6 are reported. Fig. 6 depicts, for each of the compared injection schemes, the average Packet Delivery Ratio (PDR) resulting from injecting a small number $n=3$ of message copies in the VANET. The PDR for a given injection is measured as the ratio between the vehicles receiving the message (directly or through V2V multi-hop broadcast retransmissions) and the total number of vehicles in the scenario at that moment. The PDR is then averaged over subsequent message injections. Along with the PDR, Fig 6 depicts the total UMTS uplink overhead required by the compared dissemination schemes. Besides the overhead needed to generate the VANET's connectivity characterization (Fig. 5a), the total overhead also takes into account the uplink messages that vehicles transmit to the TMC to notify when they enter and leave the target area. As shown in Fig. 6 , by only injecting 3 copies the injection schemes based on RoAHD's IRC characterization achieve a PDR of nearly $60 \%$, which is very close to the PDR that would be obtained with a perfect knowledge of vehicle positions at every moment (idealistic characterization). The delivery performance of RoAHD's IRC injections demonstrates that the connectivity characterization based on road segments' multi-hop connectivity properties is a good means to drive centralized injection strategies. In fact, injecting on connected sets of intersections having high coverage level $C L_{C S I i}$ implies targeting zones that can reliably support the multi-hop dissemination of the message, while addressing the highest number of recipient vehicles. The PDR of the dissemination based on the GPS characterization indicates that even if vehicles do not upload their positions very frequently, the TMC can retrieve a relatively precise connectivity characterization to perform good injection decisions. However, this precision is paid at the expense of a UMTS uplink overhead being 4 times bigger compared to that generated by RoAHD's IRC context characterization. Finally, the lower PDR of the random injection scheme derives from the fact that randomly selected injection vehicles may be disconnected from other vehicles of the VANET, or belong to the same CSV, and thereby prevent an effective $\mathrm{V} 2 \mathrm{~V}$ dissemination of the message.

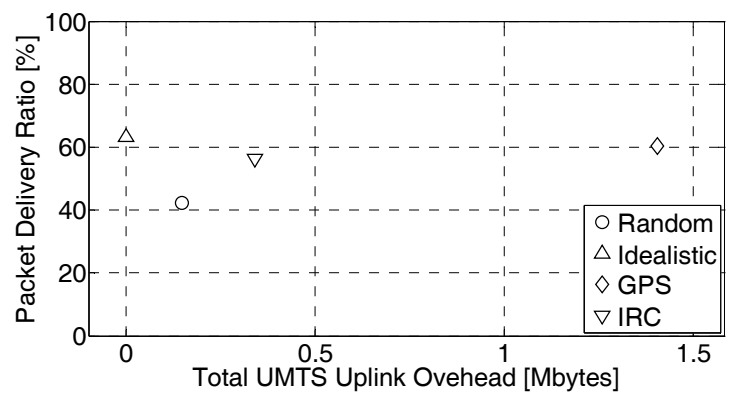

Fig. 6. PDR vs. total UMTS uplink overhead for the compared dissemination schemes.

\section{RELATED WORK}

Data dissemination through communications systems combining infrastructure-based radio technologies and adhoc networks has been recently explored in the emerging field of mobile social networks. In this context, the approaches defined in [16] and [17] opportunistically exchange in the ad-hoc network the contents received by the infrastructure-based technology with the common objective of increasing the overall system capacity while relieving the traffic on the infrastructure-based communications system.

In the context of cooperative ITS systems, hybrid dissemination approaches are investigated in a limited number of works. The authors of [18] propose STEID, an emergency dissemination protocol integrating cellular technology with clusters of vehicles in the VANET. In each cluster, a clusterhead periodically downloads emergency messages, and disseminates them in its cluster. Compared to solutions that only use the cellular system, STEID is proved to reduce the cellular channel load. However, the control traffic needed in the VANET to form, update, and maintain clusters is not evaluated. A similar hybrid system architecture to that defined in [18] is used in [19] to investigate situations in which vehicles may not initially want to cooperate in the formation of clusters and in the dissemination of messages received from the cellular base station. To give vehicles an incentive to form clusters, the authors propose a solution based on the coalition game theory. The incentive is measured as the utility (in terms of probability of successful transmissions and data rate) gained by vehicles if cooperating in the message dissemination. In [20], a "cross-network information dissemination" approach is presented. Based on this approach, a reduced percentage of vehicle acts as VANET's gateways for traffic alerts coming from the UMTS network. The authors envision estimating the vehicular spatial density by existing UMTS traffic data collection mechanisms. This estimation is then communicated to gateway nodes for them to optimize multihop broadcasting protocols in the VANET. Although very interesting for its design, [20] does not specify how the collected data traffic can be processed to derive useful inputs for the V2V broadcasting protocols, and does not explain how gateway nodes can be selected to ensure overall dissemination system's performance. Differently from the previous schemes, Push \& Track [8] aims at delivering messages to all the vehicles in a relevance area by a servicedependent expiration deadline. For this purpose, the UMTS network injects message copies to individual vehicles for them to start disseminating in the VANET using opportunistic communications. A feedback loop in which all the vehicles acknowledge receptions through UMTS uplinks permits computing how many message copies have still to be injected and to which vehicle. Through this approach, [8] demonstrated that the highest delivery ratios can be achieved by just injecting a very limited number of copies whenever a VANET's V2V connectivity characterization is hold. Inspired by these results, RoAHD defines a new framework for VANET global connectivity characterization and exploitation that requires no feedbacks and presents lower 
channel costs on the adopted communications technologies. RoAHD's connectivity characterization permits injecting message copies over the most multi-hop connected road segments. In this way, message copies can be multi-hop rebroadcasted to simultaneously address the majority of vehicles. Through this approach, good delivery ratios are obtained although not using, differently from Push \& Track, opportunistic retransmissions aimed at reaching isolated nodes that are not initially addressed by injections.

\section{CONCLUSION}

This paper has introduced RoAHD, a hybrid V2X dissemination scheme for the centralized dissemination of traffic efficiency messages in vehicular networks. RoAHD exploits in a combined way the extended coverage capabilities of cellular systems and the multi-hop communications potential of vehicular ad-hoc networks. As argued, this integration permits to overcome the inefficiencies that could rise from the isolated use of one of these two technologies. The presented simulation results demonstrate that the knowledge of a global V2V connectivity characterization benefits the delivery performance of hybrid dissemination schemes like RoAHD. Thanks to the adoption of inexpensive information concerning the multi-hop connectivity properties of entire road segments, this context characterization can be achieved by RoAHD with much less cellular channel cost than traditional approaches based on uploaded vehicle GPS positions. By exploiting the achieved connectivity context, RoAHD has been demonstrated to be capable to operate smart injection decisions, which allow achieving good delivery ratios with a limited number of injected messages.

Since this work is aimed at evaluating the capability of RoAHD to inject messages where the VANET can support reliable multi-hop retransmissions, opportunistic forwarding mechanisms are not considered. As future work, we foresee investigating how opportunistic and multi-hop broadcast retransmissions can be integrated to improve RoAHD's performance. The effects deriving from the presence of fixed ITS G5-based roadside units in the overall RoAHD's framework will be also studied.

\section{ACKNOWLEDGMENT}

This work has been partly supported by the Spanish Ministry of Economy and Competitiveness and FEDER funds under the Opportunities project (TEC2011-26109), by the Spanish Ministry of Industry, Tourism and Trade under the COMOSEF project (TSI-020401-2012-5, Celtic proposal CPP2011/2-2), and by the EU FP7 MOTO project (317959).

\section{REFERENCES}

[1] IEEE Standards Association, "Wireless LAN Medium Access Control (MAC) and Physical Layer (PHY) Specifications Amendment 6: Wireless Access in Vehicular Environments", Standard IEEE $802.11 \mathrm{p}-2010,2010$

[2] ETSI TC ITS, "Intelligent Transport Systems (ITS); European profile standard on the physical and medium access layer of $5 \mathrm{GHz}$ ITS", Standard ETSI ES 202663 v1.1.0, 2010.
[3] ETSI TC ITS, "Intelligent Transport Systems (ITS); Communications Architecture", Standard ETSI EN 320 665, v 1.1.1, 2010

[4] I. Leontiadis and C. Mascolo, "Opportunistic spatio-temporal dissemination system for vehicular networks", Proc. of the 1st International MobiSys workshop on Mobile opportunistic networking (MobiOpp '07), pp. 39-46, June 2007

[5] F.J. Ros, P.M. Ruiz, I. Stojmenovic, "Acknowledgment-Based Broadcast Protocol for Reliable and Efficient Data Dissemination in Vehicular Ad Hoc Networks", IEEE Transactions on Mobile Computing, Vol. 11, n. 1, pp. 33-46, Jan. 2012

[6] I. Leontiadis, P. Costa, C. Mascolo, "Persistent content-based information dissemination in hybrid vehicular networks", Proc. Of the IEEE International Conference on Pervasive Computing and Communications (PerCom 2009), pp.1-10, March 2009

[7] D. Borsetti and J. Gozalvez, "Infrastructure-assisted geo-routing for cooperative vehicular networks", Proc. Of the IEEE Vehicular Networking Conference 2010 (VNC 2010), pp.255-262, Dec. 2010

[8] J. Whitbeck, M. Amorim, Y. Lopez, J. Leguay, V. Conan, "Relieving the wireless infrastructure: When opportunistic networks meet guaranteed delays", Proc. Of the IEEE International Symposium on a World of Wireless, Mobile and Multimedia Networks (WoWMoM), pp.1-10, June 2011

[9] I. Lequerica, P.M. Ruiz, V. Cabrera, "Improvement of vehicular communications by using $3 \mathrm{G}$ capabilities to disseminate control information", IEEE Network, Vol. 24, n. 1, pp. 32-38, Feb. 2010

[10] A. Bazzi, B.M. Masini, O. Andrisano, "On the impact of real time data acquisition from vehicles through UMTS", Proc. Of IEEE 21 st International Symposium on Personal Indoor and Mobile Radio Communications (PIMRC), pp.1917-1922, Sept. 2010

[11] M. Rondinone and J. Gozalvez, "Distributed and Real Time Communications Road Connectivity Discovery through Vehicular Ad-hoc Networks", Proc. of the 13th International IEEE Conference on Intelligent Transportation Systems, pp.1079-1084, Sept. 2010

[12] M. Rondinone and J. Gozalvez, "Contention-based Forwarding with Multi-hop Connectivity Awareness in Vehicular Ad-hoc Networks", Elsevier Computer Networks, Available online March 2013, DOI: 10.1016/j.comnet.2013.02.019

[13] M. Rondinone et al., "iTETRIS: a modular simulation platform for the large scale evaluation of cooperative ITS applications", Elsevier Simulation Modelling Practice and Theory, Vol. 34, pp. 99-125, May 2013

[14] WINNER Consortium, "D1.1.2. WINNER II channel models", WINNER European Research project Public Deliverable, Sept. 2007

[15] ETSI, "Universal Mobile Telecommunications System (UMTS); LTE; Common test environments for User Equipment (UE); Conformance testing", 3GPP TS 34.108 v. 11.4.0 Rel. 11, Feb. 2013.

[16] S. Ioannidis, A. Chaintreau, L. Massoulie, "Optimal and scalable distribution of content updates over a mobile social network", Proc. Of IEEE INFOCOM, pp.1422-1430, Apr. 2009

[17] B. Han, P. Hui, V. Kumar, M. Marathe, J. Shao, A. Srinivasan, "Mobile Data Offloading through Opportunistic Communications and Social Participation", IEEE Transactions on Mobile Computing, Vol.11, n..5, pp.821-834, May 2012

[18] J. Nzouonta and C. Borcea, "STEID: A Protocol for Emergency Information Dissemination in Vehicular Networks", New Jersey Institute of Technology Technical Report, 2006

[19] Y. Li, K. Ying, P. Cheng, H. Yu, H. Luo, "Cooperative data dissemination in cellular-VANET heterogeneous wireless networks", Proc. Of the 4th International High Speed Intelligent Communication Forum (HSIC), pp.1-4, May 2012

[20] G. Ferrari, S. Busanelli, N. Iotti, Y. Kaplan, "Cross-network information dissemination in VANETs", Proc. Of the 11 th International Conference on ITS Telecommunications (ITST), pp. 351-356, Aug. 2011 